\title{
Nitrogen release properties of urea-kaolinite controlled release fertilizer with chitosan binder
}

\begin{abstract}
To reduce environmental and economic concerns of feeding crops with traditional fertilizers, a new type of controlled release fertilizer was prepared. In this study, ureaï kaolinite was mixed with chitosan as a binder and then was granulated. The granules were incubated in water at room temperature for 30 days and urea release was measured in 5-days intervals using UV/Vis technique and through diacetylmonoxime (DAM) calorimetric method. In this study, different binder concentration was investigated. The results of UVï Vis spectroscopy illustrated that by increasing the chitosan concentration, nitrogen release decreased from 41.23 to $25.25 \%$ after $24 \mathrm{~h}$ and from 77.31 to $59.27 \%$ after 30 days incubation in water. By doing this, the prepared controlled release fertilizer (CRF) behaved according to the standard for CRFs. Compressive stress at break was measured for granules with different size and various concentration of chitosan. Results show that the force required to crush the granules is a function of the granule diameter and binder concentration.
\end{abstract}

Keyword: Chitosan binder; Urea; Kaolinite; Controlled release fertilizer; Nitrogen release; Compressive stress at break 